International Journal of Pure and Applied Mathematics

Volume 98 No. 3 2015, 399-411

ISSN: 1311-8080 (printed version); ISSN: 1314-3395 (on-line version)

url: http://www.ijpam.eu

doi: http://dx.doi.org/10.12732/ijpam.v98i3.12

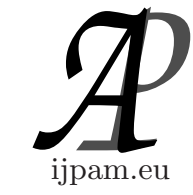

\title{
EXPONENTIAL DELAY POLYNOMIALS OF DISCRETE DELAY DETERMINANT WITH BLOOD CELLS TRANSFORMATION
}

\author{
M. Vidhya ${ }^{1}$, S. Balamuralitharan ${ }^{2}$, A. Govindarajan ${ }^{3}$ \\ ${ }^{1}$ Department of Mathematics \\ Sathyabama University \\ Chemanchery, Sholinganallur, 600 119, Tamil Nadu, INDIA \\ ${ }^{2,3}$ Department of Mathematics \\ Faculty of Engineering and Technology \\ SRM University \\ Kattankulathur, 603 202, Tamil Nadu, INDIA
}

\begin{abstract}
The purpose of this paper is to investigate the exponential delay polynomials of discrete delay determinant with blood cells transformation. By using symmetric functions, a number of new delay criteria are provided. It is shown that a discrete system with delays can be stabilized by shift operators even if it may be stable itself. The duality between the substractions on the set of biexponents and the movements of cells in the delay will be extensively employed throughout this paper, explicitly or implicitly. Some theorems and examples are also presented to illustrate the effectiveness and the superiority of the obtained results.
\end{abstract}

AMS Subject Classification: 11L07, 05E05, 05E10, 47B37

Key Words: delay determinant, exponential delay, symmetric function, shift operator

\section{Introduction}

For the recent contribution, we refer the reader to [1-16] and the references

Received: December 12, 2014

(C) 2015 Academic Publications, Ltd.

$\S$ Correspondence author url: www.acadpubl.eu 
cited therein. Delay models are becoming more common, appearing in many branches of biological modeling [1]. They have been used for describing several aspects of infectious disease dynamics: primary infection, drug therapy and immune response, to name a few. Delays have also appeared in the study of chemostat models, circadian rhythms, epidemiology, the respiratory system, tumor growth and neural networks. We have developed a method of reducing such questions to the problem of determining the existence or otherwise of cells counting determinant of a exponential polynomials. Ideally, a better understanding of the symmetric analytic theorems at work here would lead to easier determination of the existence or otherwise of blood cells partition, at least in the case of a system of only discrete delay equations. We hope that continued study of the question of blood cells count will lead to steps in the direction of such theorems for delay models. Because numerical methods for both cardinality and symmetric functions are intended for problems with solutions that have several circled blood cells partition, cells shift in low-order transformation require special attention. All of the models studied incorporate a discrete delays $[6]$.

\section{Preliminaries}

Definition 2.1. A discrete delay is a finite subset of $\mathbb{N} \times \mathbb{N}$. For $\mu_{1} \geq$ $\mu_{2} \geq \cdots \geq \mu_{k}>0$, we say that $\mu=\left(\mu_{1}, \mu_{2}, \ldots, \mu_{k}\right)$ is a blood cells partition of $n$ if $|\mu|=\mu_{1}+\cdots+\mu_{k}$ equals $n$. We associate to a cells partition $\mu$ its blood cells counting $\left\{(i, j): 0 \leq i \leq k-1,0 \leq j \leq \mu_{i+1}-1\right\}$ and we shall use the symbol $\mu$ for both the blood cells partition and its cells counting [2],[3].

Definition 2.2. Given a delay $\tau=\left\{\left(p_{1}, q_{1}\right),\left(p_{2}, q_{2}\right), \ldots,\left(p_{n}, q_{n}\right)\right\}$ with $n$ cells we define the delay determinant

$$
\Delta_{\tau}(X, Y)=\operatorname{det}\left(x_{i}^{p_{j}} y_{i}^{q_{j}}\right)_{1 \leq i, j \leq n} .
$$

The polynomial $\Delta_{\tau}(X, Y) \in \mathbb{Q}\left[X_{n}, Y_{n}\right]=\mathbb{Q}[X, Y]$ (with $m=n$, the number of cells in the delay $\tau$ ) is different from zero only if the delay $\tau$ consists of $n$ distinct cells in the positive quadrant. In this case $\Delta_{\tau}$ is homogeneous of degree $|p|=p_{1}+\cdots+p_{n}$ in $X$ and of degree $|q|=q_{1}+\cdots+q_{n}$ in $Y$. To insure that this definition associates a unique determinant to $\tau$ we require that the list of discrete blood cells is given with respect to the order with priority to the second entry that is to say:

$$
\left(p_{1}, q_{1}\right)<\left(p_{2}, q_{2}\right) \quad \Longleftrightarrow \quad q_{1}<q_{2} \text { or } \quad\left[q_{1}=q_{2} \text { and } p_{1}<p_{2}\right] .
$$


Definition 2.3. For a polynomial $P(Z) \in \mathbb{Q}[Z]$, the vector space spanned by all the partial derivatives of $P$ of all orders is denoted $\tau_{\partial}[P]$, i.e.:

$$
\tau_{\partial}[P]=\mathbb{Q}[\partial Z],
$$

where for a polynomial $Q$ in $\mathbb{Q}[Z], Q(\partial)=Q(\partial Z)$ denotes the differential operator obtained by substituting $x_{i}$ and $y_{i}$ respectively by $\partial x_{i}$ and $\partial y_{i}$ in the expression of $Q$. Next we define

$$
V_{\tau}=\tau_{\partial}\left[\Delta_{\tau}(X, Y)\right]
$$

the vector space associated to the discrete delay $\tau[5]$.

Definition 2.4. For each integer $r \geq 1$, the $r$-th power sum $P_{r}(X)$ is defined by

$$
P_{r}(X)=\sum x_{i}^{r}
$$

For each integer $r \geq 0$, the $r$-th elementary symmetric function $e_{r}(X)$ is the sum of all products of $r$ distinct variables $x_{i}$, so that $e_{0}=1$ and for $r \geq 1$ :

$$
e_{r}(X)=\sum_{i_{1}<\cdots<i_{r}} x_{i_{1}} \cdots x_{i_{r}} .
$$

For each integer $r \geq 0$, the $r$-th complete symmetric function $h_{r}(X)$ is the sum of all monomials of total degree $r$ in the variables $x_{i}$, so that:

$$
h_{r}(X)=\sum_{i_{1} \leq \cdots \leq i_{r}} x_{i_{1}} \cdots x_{i_{r}} .
$$

Definition 2.5. Let $V$ be a vector subspace of $\mathbb{Q}[Z]$ where $Z$ is a subvariable of $\left(X_{n+k}, Y_{n+k}\right)$. We define its exponential delay as the following delay:

$$
\exp (\tau)=\{P \in \mathbb{Q}[Z]: \forall Q \in V, P(\partial) Q=0\} .
$$

If $P(\partial) Q=0$, we shall say that $P$ "delete" $Q$.

If $V=\rceil x p(\tau)_{\partial}[P]$ then we denote its exponential delay simply by $\exp (\tau)_{P}$. In the case of $V_{i, j}^{k}$ we denote $\exp (\tau)_{V_{i, j}^{k}}$ by $\exp (\tau)_{i, j}^{k}$.

Definition 2.6. Let $V=V(X, Y)$ be a subspace of $\mathbb{Q}[X, Y]$. Then we denote by $V(X)$ the subspace of $V$ consisting of elements of $0 Y$-degree. We also denote the exponential delay of $V(X)$ by $\exp (\tau) V(X)$.

The goal of this section is to obtain an explicit basis for $V_{i, j}^{k}(X)$, the subspace of $V_{i, j}^{k}(X, Y)$ of elements of $0 Y$-degree [7]. 


\section{The Symmetric Operators}

We state the following propositions for $X$-shifts. Of course similar results also hold for $Y$-shifts. The only difference concerns the signs.

Proposition 3.1. Let $\tau$ be a discrete delay. Then for any integer $k \geq 1$ we have

$$
P_{k}(\partial X) \Delta_{\tau}(X, Y)=\sum_{i=1}^{n} \pm \epsilon\left(\tau, P_{k}(i ; L)\right) \Delta_{P_{k}(i ; \tau)}(X, Y)
$$

where $P_{k}(i ; \tau)$ is the delay obtained by replacing the $i$-th biexponent $\left(p_{i}, q_{i}\right)$ by $\left(p_{i}-k, q_{i}\right)$ and the coefficient $\epsilon\left(\tau, P_{k}(i ; \tau)\right)$ is a positive integer. The sign in 3.1 is the sign of the permutation that reorders the obtained biexponents with respect to the cells counting [8].

Proof. This is a particular case of Proposition I.1 in [11], but we shall give here a simple proof because some ingredients will be useful later.

If the delay $\tau$ consists of the cell columns $\tau=\left\{\left(p_{1}, q_{1}\right), \ldots,\left(p_{n}, q_{n}\right)\right\}$, we can develop the delay determinant $\Delta_{\tau}$ with respect to the $j$-th column and write:

$$
\Delta_{\tau}(X, Y)=\sum_{i=1}^{n} x_{j}^{p_{i}} y_{j}^{q_{i}} \cdot A_{i, j}
$$

where $A_{i, j}$ denotes the cofactor $(i, j)$. Let us remark that this cofactor is a polynomial where the variable $x_{j}$ does not appear. We obtain:

$$
\partial x_{j}^{k} \Delta_{\tau}(X, Y)=\sum_{i=1}^{n} c_{i}^{k} x_{j}^{p_{i}-k} y_{j}^{q_{i}} \cdot A_{i, j}
$$

where $c_{i}^{k}=p_{i}\left(p_{i}-1\right) \cdots\left(p_{i}-k+1\right)$. Next we sum 3.3 over $j$ to get:

$$
P_{k}(\partial X) \Delta_{\tau}(X, Y)=\sum_{i=1}^{n} c_{i}^{k} \sum_{j=1}^{n} x_{j}^{p_{i}-k} y_{j}^{q_{i}} \cdot A_{i, j}
$$

Thus we obtain 3.1 by recognizing in 3.4 the development of $\Delta_{P_{k}(i ; \tau)}$. As a biproduct we observe that $\epsilon\left(\tau, P_{k}(i ; \tau)\right)=c_{i}^{k}=p_{i}\left(p_{i}-1\right) \cdots\left(p_{i}-k+1\right)$ and that this coefficient does not depend on the symmetric operator $P_{k}$.

Proposition 3.2. Let $\tau$ be a discrete delay. Then for any integer $k \geq 1$ we have

$$
e_{k}(\partial X) \Delta_{\tau}(X, Y)=\sum_{1 \leq i_{1}<i_{2}<\cdots<i_{k} \leq n} \epsilon\left(\tau, e_{k}\left(i_{1}, \ldots, i_{k} ; \tau\right)\right) \Delta_{e_{k}\left(i_{1}, \ldots, i_{k} ; \tau\right)}(X, Y)
$$


where $e_{k}\left(i_{1}, \ldots, i_{k} ; \tau\right)$ is the delay of blood cells obtained by replacing the biexponents $\left(p_{i_{1}}, q_{i_{1}}\right), \ldots,\left(p_{i_{k}}, q_{i_{k}}\right)$ by $\left(p_{i_{1}}-1, q_{i_{1}}\right), \ldots,\left(p_{i_{k}}-1, q_{i_{k}}\right)$ and where the coefficient $\epsilon\left(\tau, e_{k}\left(i_{1}, \ldots, i_{k} ; \tau\right)\right)$ is a positive integer.

Proof. The proof is almost the same as for the previous proposition. We write

$$
e_{k}(X)=\sum_{1 \leq j_{1}<\cdots<j_{k} \leq n} x_{j_{1}} \ldots x_{j_{k}}
$$

We develop the determinantal delay form of $\Delta_{\tau}$ with respect to the columns $j_{1}, \ldots, j_{k}$ to obtain the following expression where $\Delta_{\tau}^{i_{1}, \ldots, i_{k}}$ denotes the delay determinant relative to the biexponents $i_{1}, \ldots, i_{k}$ of $\tau$ and $A_{i_{1}, \ldots, i_{k} ; j_{1}, \ldots, j_{k}}$ the cofactor:

$$
\Delta_{\tau}=\sum_{1 \leq i_{1}<\cdots<i_{k} \leq n} \Delta_{\tau}^{i_{1}, \ldots, i_{k}}\left(x_{j_{1}}, \ldots, x_{j_{k}}\right) A_{i_{1}, \ldots, i_{k} ; j_{1}, \ldots, j_{k}} .
$$

Next we derive 3.7 to obtain

$$
\begin{aligned}
& \partial\left(x_{j_{1}} \ldots x_{j_{k}}\right) \Delta_{\tau}=\sum_{1 \leq i_{1}<\cdots<i_{k} \leq n}\left(c_{i_{1}, \ldots, i_{k} ; j_{1}, \ldots, j_{k}} \Delta_{e_{k}\left(i_{1}, \ldots, i_{k} ; \tau\right)}^{i_{1}, \ldots, i_{k}}\left(x_{j_{1}}, \ldots, x_{j_{k}}\right)\right. \\
& \left.\times A_{i_{1}, \ldots, i_{k} ; j_{1}, \ldots, j_{k}}\right),
\end{aligned}
$$

where $c_{i_{1}, \ldots, i_{k} ; j_{1}, \ldots, j_{k}}$ is a positive integer. We see that $c_{i_{1}, \ldots, i_{k} ; j_{1}, \ldots, j_{k}}$ is equal to $p_{i_{1}} \cdots p_{i_{k}}$ and thus does not depend on $j_{1}, \ldots, j_{k}$. Therefore we can omit the subscript $j_{1}, \ldots, j_{k}$. Thus we get

$$
\begin{gathered}
e_{k}(\partial X) \Delta_{\tau}=\sum_{\left(1 \leq i_{1}<\cdots<i_{k} \leq n\right)} \sum_{\left(1 \leq j_{1}<\cdots<j_{k} \leq n\right)}\left(c_{i_{1}, \ldots, i_{k}} \Delta_{e_{k}\left(i_{1}, \ldots, i_{k} ; L\right)}^{i_{1}, \ldots, i_{k}}\left(x_{j_{1}}, \ldots, x_{j_{k}}\right)\right. \\
\left.\times A_{i_{1}, \ldots, i_{k} ; j_{1}, \ldots, j_{k}}\right) .
\end{gathered}
$$

By recognizing in 3.9 the development of $\Delta_{e_{k}\left(i_{1}, \ldots, i_{k} ; \tau\right)}$, we finally obtain the expected formula. The sign in front of the coefficient $\epsilon\left(e_{k} ; i_{1}, \ldots, i_{k} ; \tau\right)$ should be the sign of the permutation and combination that reorders the obtained biexponents in increasing order.

\section{Main Results}

Theorem 4.1. The symmetric operators are also useful to reduce the sum defining $V_{i, j}^{k}$ [9]. In the special case of one hole, it is now easy to see that

$$
V_{i, j}^{1}=V_{\mu / i, j} .
$$


Indeed we have that for any integer $k$ and $l$

$$
e_{k}(\partial X) e_{l}(\partial Y) \Delta_{\mu / i, j}=c \cdot \Delta_{\mu / i+k, j+l},
$$

with $c$ an integer different from zero. This implies $V_{i, j}^{1} \subseteq V_{\mu / i, j}$, and the reverse inclusion is obvious.

In the particular case of two holes, let $k$ and $l$ be positive integers and let us use the following notations: for two blood cells $h_{1}$ and $h_{2}, \epsilon_{h_{1}, h_{2}}^{i}=$ $\epsilon\left(\mu /\{(i, j),(i+1, j)\}, \mu /\left\{h_{1}, h_{2}\right\}\right)$ and $\epsilon_{h_{1}, h_{2}}^{j}=\epsilon\left(\mu /\{(i, j),(i, j+1)\}, \mu /\left\{h_{1}, h_{2}\right\}\right)$. If we are careful of the different signs by applying Propositions 3.1 and 3.2, then we get the following identities

$$
\begin{gathered}
P_{l}(\partial Y) e_{k-1}(\partial X) \Delta_{\mu /\{(i, j),(i+1, j)\}}=P_{l}(\partial Y)\left(\epsilon_{(i, j),(i+k, j)}^{i} \Delta_{\mu /\{(i, j),(i+k, j)\}}\right) \\
=(-1)^{b+h} \epsilon_{(i+k, j),(i, j+l)}^{i} \Delta_{\mu /\{(i+k, j),(i, j+l)\}} \\
+(-1)^{b+v+1} \epsilon_{(i, j),(i+k, j+l)}^{i} \Delta_{\mu /\{(i, j),(i+k, j+l)\}} \\
=(-1)^{b+h+1}\left((-1)^{h} \epsilon_{(i+k, j),(i, j+l)}^{j} \Delta_{\mu /\{(i+k, j),(i, j+l)\}}\right. \\
\left.+(-1)^{v} \epsilon_{(i, j),(i+k, j+l)}^{j} \Delta_{\mu /\{(i, j),(i+k, j+l)\}}\right)
\end{gathered}
$$

where $h, v$ and $b$ are respectively the numbers of cells with horizontal and vertical.

By observing that the product of the signs of the four coefficients in 4.3 and 4.4 is $(-1)^{2(2 b+2 h+v+1)+1}=(-1)$ we have that exactly three coefficients in 4.3 and 4.4 are of the same sign, whence $\Delta_{\mu /\{(i, j),(i+k, j+l)\}}$ and $\Delta_{\mu /\{(i+k, j),(i, j+l)\}}$ are in $M_{\mu /\{(i, j),(i, j+1)\}}+M_{\mu /\{(i, j),(i+1, j)\}}$.

Next, we can move simultaneously the two holes. This implies that for any couple of holes $\left(h_{1}, h_{2}\right)$ in the shadow of $(i, j)$ then $\Delta_{\mu /\left\{h_{1}, h_{2}\right\}} \in V_{\mu /\{(i, j),(i, j+1)\}}+$ $V_{\mu /\{(i, j),(i+1, j)\}}$ thus

$$
V_{i, j}^{2}=V_{\mu /\{(i, j),(i, j+1)\}}+V_{\mu /\{(i, j),(i+1, j)\}} \cdot
$$

The question of whether the obvious generalization of the previous result 4.5 is true when $k \geq 3$ appears naturally.For example it is easy to check that when $\mu=(3,2)$,

$$
\Delta_{\mu /\{(0,0),(1,0),(0,2)\}} \notin V_{\mu /\{(0,0),(1,0),(0,1)\}}+V_{\mu /\{(0,0),(0,1),(0,2)\}} .
$$

We recall the following important result ([11],[12], Proposition 1.1):

We want to prove the following proposition 


\section{Theorem 4.2.}

$\exp (\tau)_{i, j}^{k}=\bigcap_{\left(a_{1}, b_{1}\right), \ldots,\left(a_{k}, b_{k}\right)} \exp (\tau)_{\partial x_{n+1}^{a_{1}} \partial y_{n+1}^{b_{1}} \ldots \partial x_{n+k}^{a_{k}} \partial y_{n+k}^{b_{k}} \Delta_{\mu}} \cap \mathbb{Q}\left[X_{n}, Y_{n}\right] \stackrel{\text { def }}{=} \exp (\tau)$

where the intersection is over the $k$-tuples of different cells in the shadow of $(i, j)$ that we assume to be ordered [12].

Proof. Let $\left(a_{1}, b_{1}\right), \ldots\left(a_{k}, b_{k}\right)$ be $k$ cells in $S_{\mu}((i, j))$, the shadow of $(i, j)$ in $\mu$. By expanding $\Delta_{\mu}$ with respect to the last $k$ columns, we obtain:

$$
\begin{array}{r}
\Delta_{\mu}\left(X_{n+k}, Y_{n+k}\right)=\sum_{\left(a_{1}^{\prime}, b_{1}^{\prime}\right), \ldots,\left(a_{k}^{\prime}, b_{k}^{\prime}\right)} \pm \Delta_{\left\{\left(a_{1}^{\prime}, b_{1}^{\prime}\right), \ldots,\left(a_{k}^{\prime}, b_{k}^{\prime}\right)\right\}}\left(\bar{X}_{n}, \bar{Y}_{n}\right) \\
\times \Delta_{\mu /\left\{\left(a_{1}^{\prime}, b_{1}^{\prime}\right), \ldots,\left(a_{k}^{\prime}, b_{k}^{\prime}\right)\right\}}\left(X_{n}, Y_{n}\right),
\end{array}
$$

where $\bar{X}_{n}=\left\{x_{n+1}, \ldots, x_{n+k}\right\}$ and $\bar{Y}_{n}=\left\{y_{n+1}, \ldots, y_{n+k}\right\}$. Thus we get:

$$
\partial\left(x_{n+1}^{a_{1}} y_{n+1}^{b_{1}} \cdots x_{n+k}^{a_{k}} y_{n+k}^{b_{k}}\right) \Delta_{\mu}\left(X_{n+k}, Y_{n+k}\right)=c \Delta_{\mu /\left\{\left(a_{1}, b_{1}\right), \ldots,\left(a_{k}, b_{k}\right)\right\}}\left(X_{n}, Y_{n}\right)+C
$$

where $c$ is a rational constant and $C$ a linear combination with coefficients in $\mathbb{Q}\left[x_{n+1}, y_{n+1}, \ldots, x_{n+k}, y_{n+k}\right]$ of polynomials $\Delta_{\mu /\left\{\left(a_{1}^{\prime}, b_{1}^{\prime}\right), \ldots,\left(a_{k}^{\prime}, b_{k}^{\prime}\right)\right\}}$ $\left(X_{n}, Y_{n}\right)$, with:

$$
\forall 1 \leq l \leq k, \quad\left(a_{l}^{\prime}, b_{l}^{\prime}\right) \in S_{\mu}((i, j))
$$

Indeed $\Delta_{\left\{\left(a_{1}^{\prime}, b_{1}^{\prime}\right), \ldots,\left(a_{k}^{\prime}, b_{k}^{\prime}\right)\right\}}\left(\bar{X}_{n}, \bar{Y}_{n}\right)$ is not deleted by $\partial\left(x_{n+1}^{a_{1}} y_{n+1}^{b_{1}} \cdots x_{n+k}^{a_{k}} y_{n+k}^{b_{k}}\right)$ only if there exists at least a permutation $\sigma \in \mathcal{S}_{k}$, the symmetric operator on $k$ elements, such that

$$
\left(a_{\sigma(l)}^{\prime}, b_{\sigma(l)}^{\prime}\right) \in S_{\mu}\left(\left(a_{l}, b_{l}\right)\right), \quad \forall 1 \leq l \leq k .
$$

This follows easily from the definition of the $\Delta_{\left\{\left(a_{1}^{\prime}, b_{1}^{\prime}\right),\left(a_{2}^{\prime}, b_{2}^{\prime}\right), \ldots,\left(a_{k}^{\prime}, b_{k}^{\prime}\right)\right\}}$ as a determinant:

$$
\Delta_{\left\{\left(a_{1}^{\prime}, b_{1}^{\prime}\right),\left(a_{2}^{\prime}, b_{2}^{\prime}\right), \ldots,\left(a_{k}^{\prime}, b_{k}^{\prime}\right)\right\}}=\sum_{\sigma \in \mathcal{S}_{k}} \operatorname{sgn}(\sigma) x_{n+1}^{a_{\sigma(1)}^{\prime}} y_{n+1}^{b_{\sigma(1)}^{\prime}} x_{n+2}^{a_{\sigma(2)}^{\prime}} y_{n+2}^{b_{\sigma(2)}^{\prime}} \cdots x_{n+k}^{a_{\sigma(k)}^{\prime}} y_{n+k}^{b_{\sigma(k)}^{\prime}} .
$$

Taking the partial derivative $\partial\left(x_{n+1}^{a_{1}} y_{n+1}^{b_{1}} \cdots x_{n+k}^{a_{k}} y_{n+k}^{b_{k}}\right)$, we get 4.11 . For all $1 \leq$ $l \leq k$, we have $S_{\mu}\left(a_{l}, b_{l}\right) \subseteq S_{\mu}(i, j)$. Consequently $\left(a_{\sigma(l)}^{\prime}, b_{\sigma(l)}^{\prime}\right) \in S_{\mu}(i, j), \forall 1 \leq$ $l \leq k$. Because $\sigma$ is a permutation, 4.10 is now obvious. 


\section{Transformation of Blood Cells}

To illustrate the equation 4.9 , we give the following numerical example: $\mu=$ $(2,1), n=2, k=1,\left(a_{1}, b_{1}\right)=(0,0),\left(a_{2}, b_{2}\right)=(1,0)$, then

\begin{tabular}{|c|c|c|c|}
\hline 2,0 & $2,1 \diamond$ & \multicolumn{2}{|c}{} \\
\cline { 1 - 2 } 1,0 & 1,1 & \multicolumn{2}{|c}{} \\
\hline 0,0 & 0,1 & 0,2 & 0,3 \\
\hline
\end{tabular}

$$
\begin{aligned}
\partial\left(x_{4}^{0} y_{4}^{0} x_{5}^{1} y_{5}^{0}\right) \Delta_{\mu}\left(X_{3}, Y_{3}\right) & =\Delta_{\mu /\{(0,0)(1,0)\}}\left(X_{2}, Y_{2}\right)+y_{5} \Delta_{\mu /\{(0,0)(1,1)\}}\left(X_{2}, Y_{2}\right) \\
& -y_{4} \Delta_{\mu /\{(1,0)(0,1)\}}\left(X_{2}, Y_{2}\right) \\
& +\left(-x_{4} y_{5}+x_{4} y_{4}\right) \Delta_{\mu /\{(1,0)(1,1)\}}\left(X_{2}, Y_{2}\right) \\
& -y_{4}^{2} \Delta_{\mu /\{(1,0)(0,2)\}}\left(X_{2}, Y_{2}\right)+y_{4} y_{5} \Delta_{\mu /\{(0,1)(1,1)\}}\left(X_{2}, Y_{2}\right) \\
& -y_{4}^{2} y_{5} \Delta_{\mu /\{(1,1)(0,2)\}}\left(X_{2}, Y_{2}\right) .
\end{aligned}
$$

Hence we get what we want because:

- $\exp (\tau) \subseteq \exp (\tau)_{i, j}^{k}$ : let $P$ be a polynomial in $\exp (\tau)$. Since $P$ deletes the left-hand side of 4.9 , it deletes the constant term in $\mathbb{Q}\left[\bar{X}_{n}, \bar{Y}_{n}\right]$ of the lefthand side which is $\Delta_{\mu /\left\{\left(a_{1}, b_{1}\right), \ldots,\left(a_{k}, b_{k}\right)\right\}}\left(X_{n}, Y_{n}\right)$. Thus $P$ is in $\exp (\tau)_{i, j}^{k}$.

- $\exp (\tau)_{i, j}^{k} \subseteq \exp (\tau)$ : let $P$ be a polynomial in $\exp (\tau)_{i, j}^{k}$. By 4.10, $P$ deletes all the terms of the right-hand side of 4.9 ; thus it deletes the left-hand side. This implies $P \in \exp (\tau)$.

We first recall results about $V_{\mu}(X)$ the subspace of $V_{\mu}$ of elements of 0 $Y$-degree. When $\mu$ is a partition of $n$, we have

$$
\operatorname{dim} V_{\mu}(X)=n ! / \mu !
$$

where $\mu !=\mu_{1} ! \cdots \mu_{k} !$

Now let $\mu$ be a partition of $n+k$. Next we choose in the blood cells diagram $\mu, k$ cells which are simultaneously in the shadow of $(i, j)$ and such that any circled blood cell has either a cell outside the partition on its right or a circled cell (before transformation). A circled blood cell satisfying this condition is said to be "Right". We denote by $\mathcal{F}_{\mu}^{k}$ the set of the obtained objects, which we call Right blood cells. 

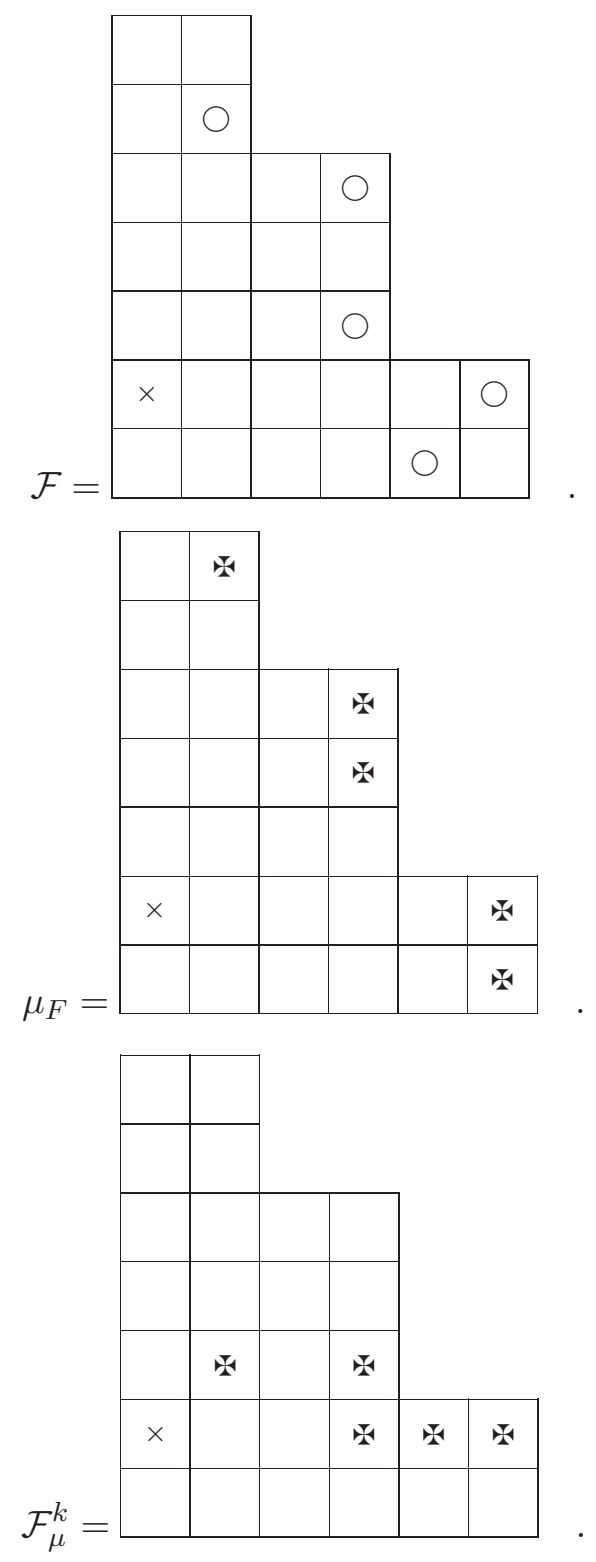

Figure 1: blood Cells-Transformation

We are now going to associate to each Right blood cells: a partition and a diagram with $k$ holes in the shadow of $(i, j)$. In this figure, the chosen blood 
cells in the Right diagram are cells with a circle, in the cell $(i, j)$ appears a $\times$ sign and the holes are as usual cells with crosses after transformation ( $\mathbf{\Psi})$. In this example $n=28$ and $k=5$. We also define a Figure $1 \mu_{F}^{k}$ with $k$ holes by proceeding as follows. We look at the columns where a circled blood cell appears. In our example we have 6 such columns. For a column $j^{\prime} \geq j$ where a circled blood cell appears, we denote by $h\left(j^{\prime}\right)$ the number of places where we could have put a circled blood cell below the lowest circled cell of this column. In our example, we have: $h(1)=0, h(2)=1, h(3)=0, \ldots, h(6)=1$. Next for any column $j^{\prime}$ with a circled blood cell, we do the following. We denote the positions of the circled cells in this column by $\left(c\left(j^{\prime}\right), j^{\prime}\right),\left(c\left(j^{\prime}\right)+a_{1}, j^{\prime}\right), \ldots,\left(c\left(j^{\prime}\right)+a_{d}, j^{\prime}\right)$, with $\left(c\left(j^{\prime}\right), j^{\prime}\right)$ the position of the lowest one, $0<a_{1}<\cdots<a_{d}$, and $d+1$ the number of circled blood cells in the column $j^{\prime}$. We then place holes in cells $\left(i+h\left(j^{\prime}\right), j^{\prime}\right),\left(i+h\left(j^{\prime}\right)+a_{1}, j^{\prime}\right), \ldots,\left(i+h\left(j^{\prime}\right)+a_{d}, j^{\prime}\right)$. Doing this for all columns gives the diagram $\mu_{F}^{k}$.

\section{An Example}

For example, given the partition $(4,2,2)$, its partition blood cell counting is

\begin{tabular}{|l|l|l|l|}
\hline 2,0 & 2,1 & \multicolumn{2}{|c}{} \\
\cline { 1 - 2 } 1,0 & 1,1 & \multicolumn{2}{|c}{} \\
\hline 0,0 & 0,1 & 0,2 & 0,3 \\
\hline
\end{tabular}

It consists of the discrete cells $\{(0,0),(1,0),(2,0),(2,1)(0,1),(1,1),(0,2),(0,3)\}$.

Let now $X=X_{m}=\left\{x_{1}, x_{2}, \ldots, x_{m}\right\}$ and $Y=Y_{m}=\left\{y_{1}, y_{2}, \ldots, y_{m}\right\}$ be two sets of $m$ variables and $\mathbb{Q}[X]=Q\left[x_{1}, x_{2}, \ldots, x_{m}\right]$ and $\mathbb{Q}[X, Y]=Q\left[x_{1}, x_{2}, \ldots, x_{m}\right.$, $\left.y_{1}, y_{2}, \ldots, y_{m}\right]$ denote respectively the cells of polynomials in $m$ and $2 m$ variables with rational coefficients. Since we have to deal with polynomials in $\mathbb{Q}[X]$ or $\mathbb{Q}[X, Y]$, we shall denote by $Z$ a subvariable of $(X, Y)$ and by $\mathbb{Q}[Z]$ the corresponding cells of polynomials.

A permutation and cobination $\sigma \in \S_{n}$ acts diagonally on a polynomial $P(X, Y) \in \mathbb{Q}\left[X_{n}, Y_{n}\right]$ as follows:

$$
\sigma P(X, Y)=P\left(x_{\sigma_{1}}, x_{\sigma_{2}}, \ldots, x_{\sigma_{n}}, y_{\sigma_{1}}, y_{\sigma_{2}}, \ldots, y_{\sigma_{n}}\right) .
$$

Under this action, $\Delta_{\tau}(X, Y)$ is clearly an alternate. It follows that for any discrete delay $\tau$ with $n$ cells, the vector space $V_{\tau}$ is an $\S_{n}$-module. Since $\Delta_{\tau}(X, Y)$ is homogeneous, this module affords a natural. 
Its interest comes in part from the fact that it gives a possible recursive approach for the $n$ ! counting, with the statement of a displacement "three term recurrence." If $\mu$ is a partition of $n+1$, we denote by $\mu / i j$ the discrete dterminant obtained by removing the cell $(i, j)$ from the partition of $\mu$. We refer to the cell $(i, j)$ as the hole of $\mu / i j$. It is counted in [2] that the number of copies of the left regular representations in $V_{\mu / i j}$ is equal to the cells counting of the $(i, j)$-shadow $\diamond$, where the shadow of a cell $(i, j)$, as shown on the figure below is: $S_{\mu}((i, j))=\left\{\left(i^{\prime}, j^{\prime}\right) \in \mu: i^{\prime} \geq i, j^{\prime} \geq j\right\}$.

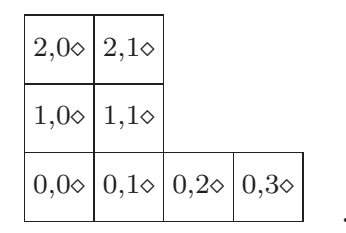

A study of the subspace $V_{\mu / i j}(X)$ of $V_{\mu / i j}$ consisting of elements of $0 Y$ degree can be found in [4], in which the corresponding "three term recursion" is proven by using the construction of explicit bases.

The aim of this article is to propose a generalization for the $n$ ! counting. The space that we consider is defined as follows. Let $\mu$ be a partition of $n+k$.

We want to describe the action of some special symmetric differential operators on the delay determinants $\Delta_{\tau}$. We recall the following definitions as stated in $[9,11]$ :

For a Figure $1 F$ in $\mathcal{F}_{\mu}^{k}$, the number of associated elements in $B_{i, j}^{k}(X)$ is equal to $\frac{n !}{r_{1} ! \cdots r_{h} !}$ where the $r_{t}$ 's are the lengths of the rows of $\mu_{F}$ because of [2] and [8]. The number of elements in $\mathcal{T}_{i, j}^{k}$ associated to $F$ is $\frac{n !}{s_{1} ! \cdots s_{h} !}$ where the $s_{t}$ 's are the lengths of the rows of $F$.

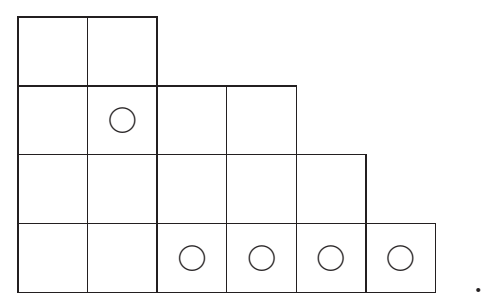




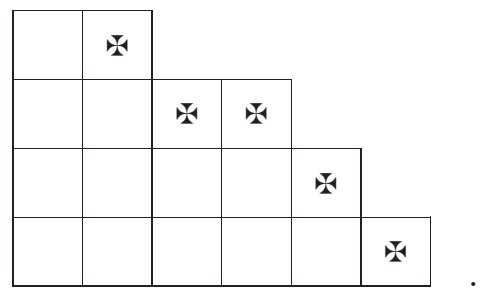

We observe that the blood cells of the circles before the transformation are $(0,3),(0,4),(0,5),(0,6),(2,2)$ and after the transformation $(0,6),(1,5),(2,3)$, $(2,4),(4,2)$. Thus the set of the lengths is unchanged.

\section{References}

[1] E. Allen, The decomposition of a bigraded left regular representation of the diagonal action of $S_{n}$, J. Comb. Theory A, 71 (1995), 97-111.

[2] Diekmann, O., Heijmans, H. J. A. M. and Thieme, H. R. (1984). On the stability of the cell size distribution. J. Math. Biology 19, 227-248

[3] J.-C. Aval, Monomial bases related to the $n$ ! conjecture, Disc. Math., 224 (2000), 15-35.

[4] Dyson, J., Villella-Bressan, R. and Webb, G. F. (1996). A singular transport equation modeling a proliferating maturity structured cell population. Can. Appl. Math. Quar. 4, 65-95

[5] F. Bergeron, N. Bergeron, A. Garsia, M. Haiman and G. Tesler, Lattice Diagram Polynomials and Extended Pieri Rules, Adv. Math., 142 (1999), 244-334.

[6] M.Buslowicz, Explicit solution of discrete-delay equations, Found. Contr. Eng., 7 (1982), 67-81.

[7] B. Liu, D. Hill, Uniform stability of large-scale delay discrete impulsive systems, International Journal of Control, 82 (2009), 228-240.

[8] I. G. Macdonald, Symmetric Functions and Hall Polynomials, Second Edition, Clarendon Press, Oxford, England (1995).

[9] Mark Haiman, Conjectures on the quotient ring by diagonal invariants, J. Algebraic Combin., 3 (1994), 17-76. 
[10] Lasota, A. and Mackey, M. C. (1984). Globally asymptotic properties of proliferating cell populations. J. Math. Biology 19, 43-62

[11] Nai Huan Jing, Vertex operators and Hall-Littlewood symmetric functions, Adv. Math., 87 (1991), 226-248.

[12] J. L. Walsh, W. E. Sewell, H. M. Elliott, On the Degree of Polynomial Approximation to Harmonic and Analytic Functions, Transactions of the American Mathematical Society, 67 (1949), 381-420.

[13] Loskot, K. (1994). Bounded solutions of partial differential equations describing interacting cell populations. Bull. Ac. Pol.: Math., 42, 315-343

[14] Y. Zhang, J. Sun, G. Feng, Impulsive control of discrete systems with time delay, IEEE Transactions on Automatic Control, 54 (2009), 830-834.

[15] W. Zhu, D. Xu, Z. Yang, Global exponential stability of impulsive delay difference equation, Applied Mathematics and Computation, 181 (2006), $65-72$.

[16] Mackey, M. C. and Rudnicki, R. (1994). Global stability in a delayed partial differential equation describing cellular replication. J. Math. Biol. 33, 89109. 
